\title{
Spectral and Photometrical Investigation of EV Lac in Different States with High Time Resolution
}

\author{
G.M. Beskin, S.N. Mitronova, I.P. Panferova
}

Special Astrophysical Observatory, Nizhnij Arkhyz, Karachaevo-Circassia, 357147 Russia

Spectroscopic and photometric monitoring of the flare star EV Lac was carried out with the 6-m telescope of the Special Astrophysical Observatory on the following 3 nights: 1993 September 10 (18:54-20:16 UT), 11 (17:46-20:03 and 21:25-22:16 UT) and 12 (19:46-20:08 and 21:23-22:21 UT).

We used the observation method described in detail by Berdyugin et al. (1994) and Alekseev et al. (1994). Observations were carried out with the 1024 channel TV scanner (Drabek et al. 1986) installed on the spectrograph SP-124 at the Nasmyth focus which covered a wavelength range $3480-5530 \AA$ with a spectral resolution $2 \AA$ per channel. Exposure times were $3-5$ minutes, the signal/noise ratio was 20 at $3500 \AA$ and 40 at $5500 \AA$ and dead time between exposures was $10-15$ sec. 92 spectra were obtained in total.

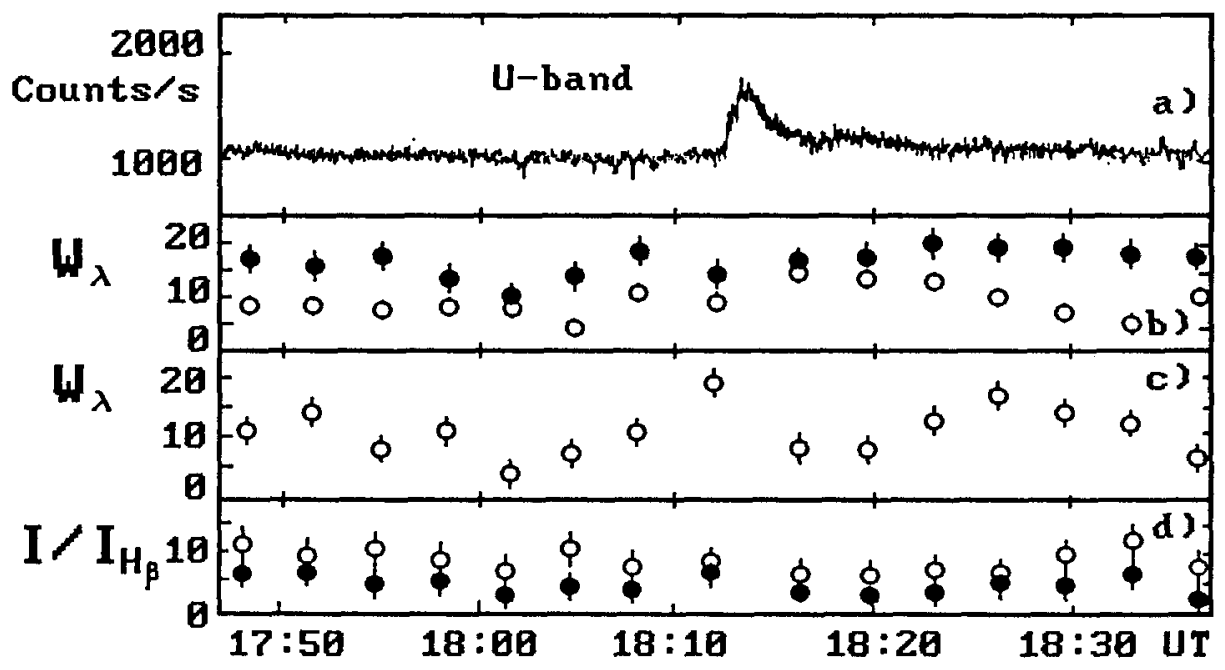

Fig. 1. Monitoring of EV Lac on the 6-m telescope SAO RAS 11/12.09.1993. (a) Light curve. Eequivalent widths of lines: (b) $\bullet-H \gamma, \circ-H \beta$; (c) $\circ-H \delta$. (d) The Balmer decrements: $\bullet-I_{H \delta} / I_{H \beta}, \circ-I_{H \gamma} / I_{H \beta}$. 
All spectroscopic observations were simultaneous with $U$ band photometry made using the MANIA (Multichannel Analysis of Nanosecond Intensity Alterations) complex. It registers the arrival time of each photon with an accuracy of 20 ns. The time resolution is $10^{-7} \mathrm{~s}$ (Beskin et al. 1982, Zhuravkov et al. 1992). 19 flares with amplitudes $\Delta U>0.1$ and 4 flares with $\Delta U>0.5$ were detected. The light curve and spectroscopic variations during a flare of $\Delta U=0.6$ on 1993 September 11 at 18:12 UT are shown in Fig. 1. An increase of equivalent widths of the lines $\mathrm{H} \beta, \mathrm{H} \gamma$ and $\mathrm{H} \delta$ before and during the photometric flare was detected (Fig. 1b,c). No such emission line variations were recorded during a fainter and shorter flare with an amplitude $\Delta U>0.4$ on 1993 September 11 at 19:50 UT. 3 segments of photometric data (Fig. 1a) were analyzed with the $y_{2}$ and $d_{2}$-function formalism (Shvartsman 1977, Plokhotnichenko 1983) to search for stochastic variability of EV Lac's brightness. The results of this statistical analysis (see Table 1) showed that there is no fast variability on time scales of 1 $\mu \mathrm{s}-10$ sec with a confidence of $99 \%$.

The value of the intensity jump near the limit of the TiO band at $4955 \AA$ and the equivalent widths of photospheric $\mathrm{CaI}$ and $\mathrm{MgH}$ lines were practically constant during the whole observation. Sporadic variations of the Balmer decrement of up to a factor 2, outside of flares, were observed on 1993 September 12.

Table 1. Upper limits for the relative power of the brightness variable component (in $\%$ ) (filling factor 0.2 , confidence probability $\geq 99 \%$ ).

\begin{tabular}{l|c|c|c|c|c}
\hline \multirow{2}{*}{$\begin{array}{c}\text { Phase of } \\
\text { brightness }\end{array}$} & \multicolumn{5}{|c}{ Time scales (s) } \\
\cline { 2 - 6 } & $10^{-6}-10^{-5}$ & $10^{-5}-10^{-3}$ & $10^{-3}-10^{-2}$ & $10^{-2}-1$ & $1-10$ \\
\hline 1. Top of flash & 55 & 34 & 14 & 6.3 & 2.2 \\
2. Tail of flash & 39 & 21 & 9 & 4.6 & 1.7 \\
3. Quiet & 37 & 23 & 9 & 4.5 & 1.5 \\
\hline
\end{tabular}

\section{References}

Alekseev I.Yu., Gershberg R.E., Iyin I.V. et al., 1994, A\&A 288, 502

Berdyugin A.V., Gershberg R.E., Iyin I.V. et al., 1994, Izv. Krymsk. Astrofiz. Obs. 89 (in press)

Beskin G.M., Neizvestny S.I., Pimonov A.A. et al., 1982, in Proc. IAU Coll. 67, Instrumentation for astronomy with large optical telescopes, C.M. Humphries (ed), Reidel, Dordrecht, p. 181

Drabek S.B., Kopylov I.M., Somov N.N., Somova T.A., 1986, Astrofiz. Issled. (Izvestiya Spets. Astrofiz. Obs.) 22, 64

Plokhotnichenko V.L., 1983, Soobshcheniya Spets. Astrofiz. Obs. 38, 29

Shvartsman V.F., 1977, Soobshcheniya Spets. Astrofiz. Obs. 19, 5

Zhuravkov A.V., Pimonov A.A., Plokhotnichenko V.L., 1992, SAO RAS Prepr. 91 